\title{
Pancreatic Cancer Progression in a Patient With Lynch Syndrome Receiving Immunotherapy: A Cautionary Tale
}

\author{
Yifan Wang, MD ${ }^{1,2,3}$; Adeline Cuggia, MSc, $\mathrm{CCGC}^{2}$; Alain Pacis, $\mathrm{PhD}^{3,4}$; Jean-Christian Boileau, MD; \\ Victoria A. Marcus, MD ${ }^{6}$; Zu-Hua Gao, MD, PhD ${ }^{2,6}$; George Chong, $\mathrm{PhD}^{7}$; \\ William D. Foulkes, MD, $\mathrm{PhD}^{2,8}$; and George Zogopoulos, MD, $\mathrm{PhD}^{1,2,3,8}$
}

\begin{abstract}
Pancreatic ductal adenocarcinomas (PDACs) with DNA mismatch repair deficiency (MMRd) respond preferentially to immune checkpoint inhibitors (ICls). However, a subset of MMRd PDACs does not respond to these agents. This report describes a patient with PDAC who experienced rapid disease progression suggestive of hyperprogressive disease. The case involved a 63-year-old man carrying a pathogenic germline PMS2 mutation who developed metastatic PDAC. His tumor showed isolated loss of PMS2 expression by immunohistochemistry $(\mathrm{IHC})$. He was treated with pembrolizumab, but his disease rapidly progressed. Whole-genome and transcriptome sequencing of a liver metastasis biopsy, acquired at disease progression, showed a retained wild-type PMS2 allele and hallmarks of microsatellite stability, including low tumor mutational burden and low MSlsensor score. PCRbased microsatellite instability (MSI) testing of the treatment-naïve tumor showed microsatellite stability. The $\mathrm{ICl}$-treated tumor had a lower density of CD8+ T-cell infiltration than the treatment-naïve tumor, which is contrary to the expected evolution with $\mathrm{ICl}$ responsiveness. Through this case and a review of the literature, we highlight the low penetrance of PMS2 germline mutations in PDAC and discuss pitfalls in ascertaining MMRd and MSI based on IHC testing alone. An orthogonal confirmatory assay is warranted in the presence of uncommon immunophenotypes, such as isolated PMS2 loss, to optimize selection of patients with PDAC for immunotherapy.
\end{abstract}

J Natl Compr Canc Netw 2021;19(8):883-887 doi: 10.6004/jnccn.2021.7049

\footnotetext{
${ }^{1}$ Department of Surgery, McGill University; ${ }^{2}$ Research Institute of the McGill University Health Centre; ${ }^{3}$ The Rosalind and Morris Goodman Cancer Research Centre, McGill University; ${ }^{4}$ Canadian Centre for Computational Genomics, McGill University and Genome Quebec Innovation Center; ${ }^{5}$ Department of Medical Oncology, Hôpital St-Eustache; ${ }^{6}$ Department of Pathology, McGill University; ${ }^{7}$ Molecular Diagnostics Laboratory, Sir Mortimer B. Davis-Jewish General Hospital; and ${ }^{8}$ Department of Human Genetics, McGill University, Montreal, Quebec, Canada.
}

Pancreatic ductal adenocarcinoma (PDAC) is a difficultto-treat malignancy, with a 5-year survival rate of $9 \%$. Treating PDAC with therapies that target specific molecular aberrations may be more effective than empirical chemotherapeutic strategies.

The DNA mismatch repair (MMR) genes (MLH1, MSH2, MSH6, PMS2) correct DNA replication errors. We and others have shown that approximately $1 \%$ of PDACs are related to MMR deficiency (MMRd). ${ }^{1}$ MMRd tumors typically arise in patients with Lynch syndrome (LS) who carry a pathogenic germline mutation in one of the MMR genes. In the absence of LS, MMRd can also be caused by biallelic somatic inactivation of an MMR gene, either by mutations or by epigenetic silencing.

MMRd tumors accumulate insertion and deletion mutations, particularly within microsatellite regions, leading to high microsatellite instability (MSI-H). This increased mutational burden generates neoantigens that are recognized by cytotoxic CD8+ T lymphocytes. ${ }^{1}$ One mechanism by which MMRd tumors evade immune-mediated killing is through upregulating immune checkpoints, such as PD-1 and CTLA-4. As a result, MMRd tumors are preferentially sensitive to immune checkpoint inhibitors (ICIs), which reactivate the antitumor immune response. Pembrolizumab (PD-1 monoclonal antibody) has shown a $62 \%$ objective response rate in MMRd/MSI-H PDAC. ${ }^{2}$

However, a subset of patients treated with ICIs experience a paradoxical phenomenon of accelerated disease progression, termed hyperprogressive disease (HPD). this report presents a patient with LS and PDAC who developed rapid disease progression while receiving pembrolizumab, with accompanying molecularly characterized longitudinal tumor biopsies.

\section{Case Report}

\section{Clinical Presentation}

A 63-year-old man of French-Canadian ancestry presented with a pancreatic head mass and multiple liver hypodensities. Biopsy of a liver mass revealed a poorly 
differentiated adenocarcinoma, consistent with metastasis from a pancreatic primary. The patient was treated with FOLFIRINOX (5-FU/folinic acid/irinotecan/oxaliplatin), but his disease progressed after 6 cycles (Figure 1A). He received gemcitabine/nab-paclitaxel as second-line treatment and experienced a partial response. However, he developed regimen-limiting peripheral neuropathy and was switched to gemcitabine monotherapy.

Immunohistochemistry (IHC) for the MMR proteins was performed on the liver biopsy, showing isolated loss of PMS2 expression, with intact nuclear expression of MLH1, MSH2, and MSH6 (Figure 2A). IHC for the MMR proteins was repeated, and isolated PMS2 loss was observed again. Because these IHC results were suggestive of LS, we performed germline testing for 86 cancer predisposition genes (Invitae Multi-Cancer Panel) using peripheral blood. Germline testing showed a pathogenic mutation in PMS2 (c.2117delA), confirming LS. Notably, the patient did not meet Bethesda guidelines for LS. Based on the loss of PMS2 expression, therapy was switched to pembrolizumab.

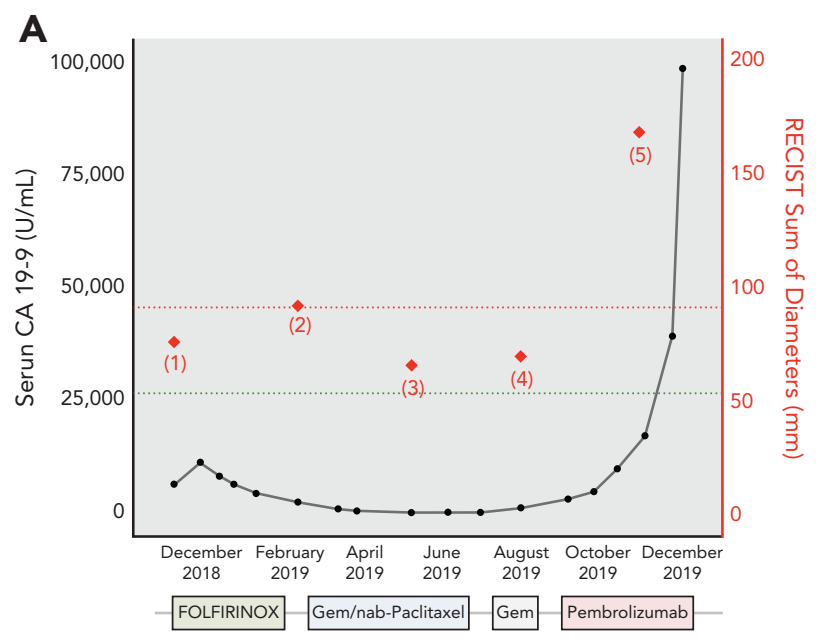

B

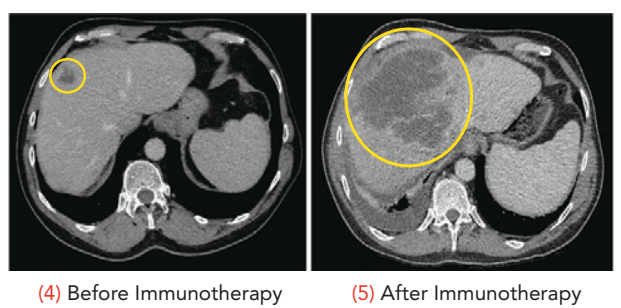

Figure 1. Clinical evolution of the patient. (A) Graph showing changes in serum CA 19-9 (black y axis and dots) and RECIST sum of diameters (red y axis and diamonds) while receiving therapy. The red and green dotted lines indicate the thresholds for progressive disease (+20\%) and partial response $(-30 \%)$, respectively. (B) CT scans obtained before initiation of pembrolizumab (left; corresponds to red diamond labeled "4" in panel A) and after 4 cycles of pembrolizumab (right; corresponds to red diamond labeled " 5 " in panel A), showing marked tumor progression consistent with hyperprogressive disease. Abbreviations: FOLFIRINOX, 5-FU/folinic acid/irinotecan/oxaliplatin; gem, gemcitabine.
Progressive Disease During Pembrolizumab Treatment After 4 cycles of pembrolizumab, restaging tomographic scans showed rapid progression of the liver metastases present at the start of immunotherapy. There was a $>2$ fold increase in the rate of tumor growth on pembrolizumab compared with that on gemcitabine/nab-paclitaxel, meeting criteria for HPD (Figure 1B).$^{3}$ In addition, there was interval appearance of innumerable new liver and lung metastases. A progression biopsy was obtained from the dominant segment 8 liver metastasis. The patient experienced rapid clinical deterioration and died 2 months later.

\section{Molecular Characterization of the Diagnostic and Progression Biopsies}

To examine the molecular mechanisms underlying the disease progression in this patient, we performed MMR IHC on the progression biopsy. Surprisingly, IHC for the MMR proteins showed intact nuclear expression of all $4 \mathrm{MMR}$ proteins (Figure 2A). Furthermore, we performed wholegenome sequencing (WGS) of the tumor and matched lymphocyte DNA to depths of $86 \times$ and $62 \times$, respectively, using methods we have described previously. ${ }^{4}$ The germline PMS2 c.2117delA mutation was identified by WGS. However, there was no evidence of a second somatic hit, and the wild-type PMS2 allele was retained (Figure 2B, C). The tumor was microsatellite stable with a low MSIsensor score (0.79). The tumor mutational burden (TMB) was 7.44 mutations per megabase, which is less than what has been reported for MMRd tumors. ${ }^{1}$ Furthermore, the COSMIC Mutational Signatures findings were 16, 5, and 12, which are not associated with MMRd. ${ }^{1}$ Common driver genes were mutated, including KRAS, TP53, and SMAD4. ${ }^{1}$ We did not identify a BRAFV600E mutation, which is associated with $\mathrm{MLHl}$ promoter hypermethylation. We also performed tumor whole-transcriptome sequencing. Wild-type PMS2 transcripts were identified. The tumor was classified as basal-like based on unsupervised clustering against 164 PDACs from the COMPASS trial (ClinicalTrials.gov identifier: NCT02750657). ${ }^{4}$

Fresh frozen tumor was not available from the initial biopsy to perform WGS. However, we evaluated the initial biopsy for MSI. After tumor macrodissection, MSI testing was performed using the clinical-grade Idylla MSI Test (Biocartis), which features a panel of 7 monomorphic microsatellite markers (ACVR2A, BTBD7, DIDO1, MRE11, RYR11, SEC31A, SULF2). The tumor was found to be microsatellite stable, with none of the 7 MSI markers showing instability.

\section{Immune Microenvironment of the Diagnostic and Progression Biopsies}

To examine differences in the tumor immune microenvironment after progression, the diagnostic and progression 


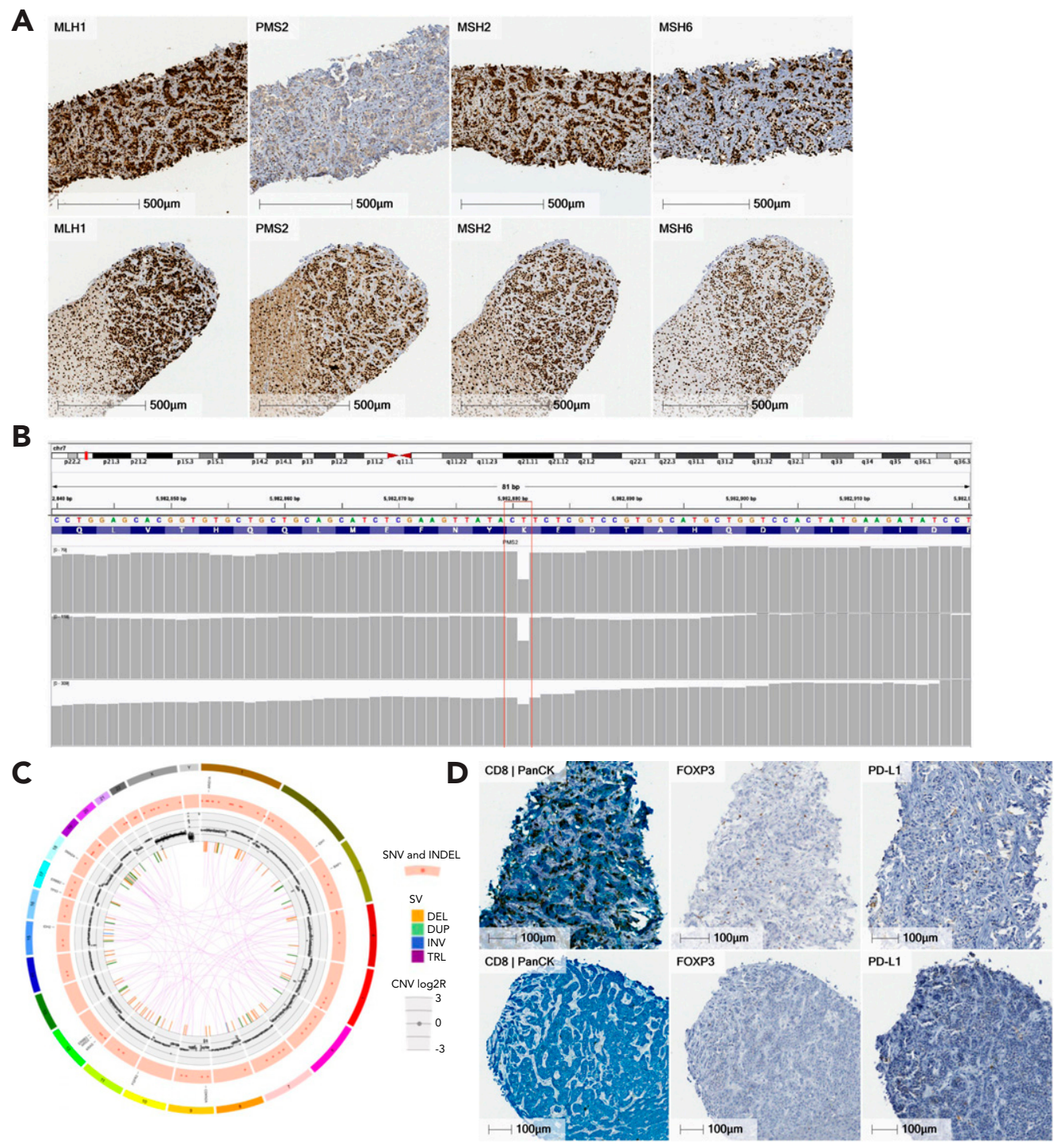

Figure 2. Molecular characterization of diagnostic and progression biopsies. (A) Mismatch repair immunohistochemistry of the diagnostic biopsy showing isolated PMS2 loss (top) and progression biopsy showing intact expression of all 4 proteins (bottom). (B) Integrative Genomics Viewer tracks of germline DNA (top), tumor DNA (middle), and tumor RNA (bottom), showing the germline PMS2 c.2117delA mutation and retention of second wild-type PMS2 allele in the tumor. (C) Circos plot depicting whole-genome sequencing of the progression biopsy. (D) Comparison of the tumor immune microenvironment of the diagnostic biopsy (top) versus the progression biopsy (bottom). In the first column, CD8 (brown) and panCK (teal) are dually stained. Abbreviations: CNV, copy number variation; DEL, deletion, DUP, duplication; INDEL, insertion or deletion mutation; INV, inversion; log2R, log 2 ratio; panCK, pan-cytokeratin; SNV, single-nucleotide variant; SV, structural variant; TRL, translocation.

biopsies were stained for CD8 (cytotoxic T lymphocytes), FOXP3 (T-regulatory lymphocytes), and PD-L1 (Figure 2D). Compared with the diagnostic biopsy, the progression biopsy had a lower density of CD8+ T cells (1.7 vs 380.4 cells $/ \mathrm{mm}^{2}$ tumor) and FOXP3+ T-regulatory cells (26.2 vs 184.7 cells/ $\mathrm{mm}^{2}$ tumor). Both biopsies were negative for PD-L1.

\section{Discussion}

This report describes a patient with LS and PDAC who had rapid disease progression while receiving pembrolizumab. The isolated loss of PMS2 expression on IHC, in the presence of a germline PMS2 mutation, was suggestive of LS-associated MMRd. However, the initial tumor was microsatellite stable on MSI testing. One explanation that could reconcile the discordant IHC and MSI findings is that a second somatic mutation in PMS2 may have been acquired as a later passenger event, rather than a causative driver. This could result in loss of protein expression while the tumor retains microsatellite stability. To this end, Alpert et $\mathrm{al}^{5}$ reported that $7.1 \%$ of colorectal cancers with isolated PMS2 loss by IHC were not MSI-H by PCR testing. In addition to the passenger mutation hypothesis, functional 
redundancy of PMS2 with MLH3 and PMS1 may play a role in rescuing MMR function.

The progression biopsy showed a retained wild-type PMS2 allele and genomic hallmarks of microsatellite stability, including a low TMB comparable to that of MMR-proficient PDACs. ${ }^{1,6}$ Furthermore, the tumor harbored typical driver mutations in KRAS, TP53, and SMAD4, which are less prevalent in MMRd PDACs. ${ }^{1}$ Together, these findings show the absence of an MMR "genomic scar" and suggest that this PDAC was unlikely MMRd or PMS2-driven, despite the initial PMS2 loss on IHC. The discrepancy between the MMR IHC results of the diagnostic biopsy and the progression biopsy may also be explained by intratumoral heterogeneity, wherein a subclonal population exhibiting focal PMS2 loss may have been sampled on the diagnostic biopsy. Indeed, patterns of MMR protein loss may be heterogeneous within a given tumor. Tachon et $\mathrm{al}^{7}$ described a case of colorectal cancer in which the primary tumor exhibited areas of MLH1/PMS2 loss, but a lymph node metastasis retained intact expression of all MMR proteins and was microsatellite stable. It is also plausible that this subclone with PMS2 loss may have been eliminated under the selective pressure of therapy. This illustrates potential pitfalls of interpreting MMR IHC based on small biopsies that may not be representative of the staining patterns across the entire tumor.

Although PMS2 is conventionally grouped with the 3 other MMR genes, PMS2 carriers have a lower risk of extracolonic tumors than carriers of other MMR gene mutations. Across 1,213 patients with PDAC, we did not identify any MMRd PDACs that were driven by a germline PMS2 mutation. ${ }^{1}$ Furthermore, PMS2 is the only MMR gene for which a germline mutation is not an indication for PDAC surveillance according to the NCCN Clinical Practice Guidelines in Oncology (NGGN Guidelines) for Genetic/Familial High-Risk Assessment: Breast, Ovarian, and Pancreatic. ${ }^{8}$ In the MSK-IMPACT experience, 2 of 3 patients with a germline PMS2 mutation developed MMR-proficient PDAC, characterized by intact PMS2 protein expression, a low TMB, and a low MSIsensor score. ${ }^{6}$ One of these patients received a combination of PD-L1 inhibitor and $\mathrm{C}-\mathrm{C}$ chemokine receptor 4 inhibitor and developed progressive disease after 3 months. These findings underscore that PDACs in individuals with PMS2 germline mutations may arise sporadically, without being driven by biallelic inactivation of PMS2. Furthermore, they highlight the rarity of bona fide PMS2-driven MMRd PDAC.

Cohen et $\mathrm{al}^{9}$ reported a cohort of patients who were assessed locally as having MMRd and MSI-H colorectal cancer and received ICIs. Of 5 patients who had primary resistance to ICIs, 3 (60\%) were found to be MMR proficient after central retesting for MMR IHC and MSI PCR. Indeed, misdiagnosis of MMRd and MSI-H status may underlie a subset of resistance to ICI. Currently, ascertainment of MMRd and MSI-H status using a single diagnostic method (IHC or PCR) is sufficient for enrollment in most ICI clinical trials. Although this practice is dictated by practical considerations, the negative impacts of a misdiagnosis cannot be understated. We propose that for any case lacking an undebatable IHC profile (ie, simultaneous loss of PMS2 and MLH1 or of MSH6 and MSH2), use of an orthogonal assay to confirm MMRd and MSI-H is warranted before initiating ICI treatment. In particular, the trigger for orthogonal confirmatory testing should include cases with isolated loss of one of the minor partners (MSH6, PMS2), which are rare immunophenotypes. This caution is particularly important in cancers that are less common in the spectrum of LS-associated malignancies, such as PDAC. ${ }^{10}$ With the advent of next-generation sequencing, genomic hallmarks of MSI can also serve as a complementary test.

There is an emerging body of literature suggesting that a subset of patients treated with ICIs experience a distinct phenomenon of accelerated tumor growth. In clinical practice, distinguishing HPD from "conventional" progressive disease is challenging, especially in the context of multiple failed lines of therapy and an aggressive malignancy such as PDAC. Several definitions of HPD have been proposed, all of which aim to show tumor growth that markedly exceeds what is expected of the natural history of the disease. Based on these definitions, the rate of HPD in patients receiving ICIs ranges between $7 \%$ and $29 \%$ across multiple tumor types. ${ }^{11,12}$ The clinical importance of the HPD phenotype is that it is associated with significantly worse survival outcomes. However, the molecular mechanisms underlying HPD remain poorly understood. Older age and presence of liver metastases have been proposed as risk factors for HPD. ${ }^{11,13}$ At a genomic level, MDM2/4 amplification, EGFR mutation, and B2M mutation have been associated with HPD, but these were not identified in our case. ${ }^{14}$ An increase in CD8+ density in ICI-treated tumors has been associated with radiologic response. ${ }^{15}$ In our case, the ICI-treated tumor displayed lower CD8+ T-lymphocyte infiltration.

\section{Conclusions}

Altogether, the absence of robust biomarkers of HPD further underscores the importance of integrating orthogonal MMRd and MSI-H testing strategies to select patients who are most likely to benefit from immunotherapy.

Submitted April 1, 2021; final revision received April 21, 2021; accepted for publication April 21, 2021.

Author contributions: Conceptualization: Wang, Zogopoulos. Methodology: Wang, Pacis, Marcus, Gao, Chong, Foulkes. Investigation: Cuggia, Boileau, 
Marcus, Gao, Chong, Foulkes, Zogopoulos. Software: Pacis. Formal analysis: Wang, Pacis. Resources: Boileau, Gao, Chong, Zogopoulos. Writing-original draft: Wang, Zogopoulos. Writing-review and editing: All authors.

Supervision: Foulkes.

Disclosures: The authors have disclosed that they have not received any financial consideration from any person or organization to support the preparation, analysis, results, or discussion of this article.
Funding: This work was supported by funding from the Fonds de Recherche du Québec-Santé, the Canadian Institutes of Health Research Vanier Canada Graduate Scholarship, and the McGill University Surgical-Scientist Program (Dr. Wang); and the Cancer Research Society and the Quebec Cancer Consortium (Dr. Zogopoulos).

Correspondence: George Zogopoulos, MD, PhD, McGill University Health Centre, 1001 Decarie Boulevard, Room EM2.3210, Montreal, Quebec, Canada H4A 3J1. Email: george.zogopoulos@mcgill.ca

\section{References}

1. Grant RC, Denroche R, Jang GH, et al. Clinical and genomic characterisation of mismatch repair deficient pancreatic adenocarcinoma [published online September 15, 2020]. Gut, doi: 10.1136/gutjnl-2020-320730

2. Le DT, Durham JN, Smith KN, et al. Mismatch repair deficiency predicts response of solid tumors to PD-1 blockade. Science 2017:357:409-413.

3. Matos I, Martin-Liberal J, García-Ruiz A, et al. Capturing hyperprogressive disease with immune-checkpoint inhibitors using RECIST 1.1 criteria. Clin Cancer Res 2020;26:1846-1855.

4. Wang Y, Park JYP, Pacis A, et al. A preclinical trial and molecularly annotated patient cohort identify predictive biomarkers in homologous recombinationdeficient pancreatic cancer. Clin Cancer Res 2020;26:5462-5476.

5. Alpert L, Pai RK, Srivastava A, et al. Colorectal carcinomas with isolated loss of PMS2 staining by immunohistochemistry. Arch Pathol Lab Med 2018; 142:523-528.

6. Hu ZI, Shia J, Stadler ZK, et al. Evaluating mismatch repair deficiency in pancreatic adenocarcinoma: challenges and recommendations. Clin Cancer Res 2018;24:1326-1336.

7. Tachon G, Frouin E, Karayan-Tapon L, et al. Heterogeneity of mismatch repair defect in colorectal cancer and its implications in clinical practice. Eur J Cancer 2018;95:112-116.

8. Daly MB, Pal T, Berry MP, et al. Genetic/Familial High-Risk Assessment: Breast, Ovarian, and Pancreatic, Version 2.2021, NCCN Clinical Practice Guidelines in Oncology. J Natl Compr Canc Netw 2021;19:77-102. To view the most recent version, visit NCCN.org
9. Cohen $\mathrm{R}$, Hain $\mathrm{E}$, Buhard $\mathrm{O}$, et al. Association of primary resistance to immune checkpoint inhibitors in metastatic colorectal cancer with misdiagnosis of microsatellite instability or mismatch repair deficiency status. JAMA Oncol 2019;5:551-555

10. Luchini C, Bibeau F, Ligtenberg MJL, et al. ESMO recommendations on microsatellite instability testing for immunotherapy in cancer, and its relationship with PD-1/PD-L1 expression and tumour mutational burden: a systematic review-based approach. Ann Oncol 2019;30:12321243.

11. Champiat $S$, Dercle $L$, Ammari $S$, et al. Hyperprogressive disease is a new pattern of progression in cancer patients treated by anti-PD-1/PD-L1. Clin Cancer Res 2017:23:1920-1928.

12. Kim CG, Kim KH, Pyo KH, et al. Hyperprogressive disease during PD-1/PDL1 blockade in patients with non-small-cell lung cancer. Ann Oncol 2019; 30:1104-1113

13. Sasaki A, Nakamura $Y$, Mishima S, et al. Predictive factors for hyperprogressive disease during nivolumab as anti-PD1 treatment in patients with advanced gastric cancer. Gastric Cancer 2019;22: 793-802.

14. Kato S, Goodman A, Walavalkar V, et al. Hyperprogressors after immunotherapy: analysis of genomic alterations associated with accelerated growth rate. Clin Cancer Res 2017;23:4242-4250.

15. Tumeh PC, Harview CL, Yearley JH, et al. PD-1 blockade induces responses by inhibiting adaptive immune resistance. Nature 2014;515: 568-571. 